CASE REPORT

\author{
V. Lai \\ Y.C. Wong \\ W.L. Poon \\ Y.P. Fu \\ T.C. Lam \\ S.C. Yuen
}

\section{Radiation-Induced Peripheral Nerve Neurofibromata in a Patient Receiving Hypofractionated Radiation Therapy}

\begin{abstract}
SUMMARY: Radiation-induced peripheral nerve tumor, in particular a benign entity such as a neurofibroma, is rare, with only a few cases being reported so far. We demonstrate a case of radiationinduced neurofibromata along the left cervical nerve roots in a man with a background of localized targeted hypofractionated radiation therapy as adjuvant treatment for left cervical nodal metastasis complicating nasopharyngeal carcinoma. The toxicity of high-dose radiation in a hypofractionated regime is also stressed.
\end{abstract}

$\mathbf{R}$ adiation-induced peripheral nerve tumors are extremely rare, and only approximately 60 cases have been reported in the literature so far. ${ }^{1}$ Sarcoma is the most common malignant radiation-induced tumor, whereas benign tumors such as neurofibroma are rare with only a few cases reported in the past, which generally occurred in patients initially irradiated at a young age, after a long postradiation interval and with longterm survival. ${ }^{1}$

With the increasing use of radiation as a therapeutic technique, emphasis has been focused on its associated short- and long-term complications, in particular with the hypofractionated regime using a higher dose of radiation. Here, we report a case of radiation-induced peripheral nerve neurofibromata in a 39-year-old man who had received hypofractionated radiation therapy to the left cervical region for cervical nodal metastasis complicating nasopharyngeal carcinoma.

\section{Case Report}

A 39-year-old man was diagnosed with nasopharyngeal carcinoma with left cervical node metastasis (T2 N2 M0) in 1985. Surgical excision of the left cervical node masses was performed, followed by conventional and hypofractionated radiation therapies targeting the nasopharynx (2.5 Gy/24 fraction of radiation dose [fr]/42 days) and left neck (5.6 Gy/7 fr/49 days) respectively. The disease had been in remission since this treatment, and he remained well and asymptomatic for 9 years.

He then had insidious onset of left-shoulder weakness since early 2004, which became worse by late 2005, with development of numbness and paraesthesia, subsequently progressing to involve the left forearm. There was no preceding injury or viral illness. Physical examination revealed muscle wasting involving the left deltoid and biceps brachialis muscles. Tendon reflexes were absent in the left upper limb. Power was recorded as $1 / 5$ only along the myotome of the left C5-C7. The clinical images suggested a lower motor neuron lesion involving the left $\mathrm{C} 5-\mathrm{C} 7$ nerve roots, which was tentatively treated as postradiation neuritis.

MR imaging of the left brachial plexus was performed initially in

Received April 15, 2008; accepted after revision May 14

From the Departments of Radiology (V.L., Y.C.W., W.L.P), Medicine and Geriatrics (Y.P.F.), Clinical Oncology (T.C.L.), and Neurosurgery (S.C.Y.), Tuen Mun Hospital, Tuen Mun, Hong Kong, China.

Please address correspondence to Vincent Lai, MD, Diagnostic Radiology, Tuen Mun Hospital, Tuen Mun N.T., Hong Kong, China; e-mail: vincentlai@hkcr.org

DOI 10.3174/ajnr.A1219
November 2005, showing swollen left C4-C8 nerve roots with abnormal T2-weighted hyperintense signal-intensity changes (Fig $1 A$ ) and contrast enhancement (Fig $1 B$ ). The results of a blood test were unremarkable. The patient was given a course of steroids, but no significant clinical improvement was observed afterward. Follow-up MR imaging in May 2006 showed persistent thickened enhancing left C4-C8 nerve roots, showing a slight interval increase in size but of similar extent otherwise (Fig 2). An electromyogram was also obtained, and the findings were suggestive of postirradiation brachial plexopathies.

Prednisolone and a course of intravenous immunoglobulin were given, but again, clinical symptoms remained the same. By early 2007, his symptoms became worse, and he developed complete paralysis of the left upper limb and severe neuralgia, mainly along the myotome of C4-C7. Further follow-up MR imaging performed in March 2007 (16 months after the initial scanning) showed masslike enlargement of the left C4-C8 nerve roots (Fig 3A). In particular, there was intraspinal extension at the level of $\mathrm{C} 6$ with cord compression (Fig $3 B$ ). Findings were compatible with neurogenic tumor.

Laminectomy and surgical excision of the masses were subsequently performed. Histology confirmed the masses to be neurofibroma with positive $\mathrm{S} 100$ protein staining and no evidence of nuclear pleomorphism or mitosis (Fig $4 A,-B$ ). In view of their location and history of irradiation, overall findings were suggestive of radiationinduced neurofibromata along the left $\mathrm{C} 4-\mathrm{C} 8$ nerve roots.

\section{Discussion}

Radiation-induced peripheral nerve tumors are a rare, yet known complication of radiation therapy. Benign tumors such as neurofibromatas are even rarer, with only a few cases reported, whereas malignant peripheral nerve sheath tumors constitute most nerve tumors. ${ }^{1}$ These are a late complication occurring years after completion of treatment, varying from 5 to 31 years. The nature and extent of radiation-induced tumor depend on several factors, including total dose to and volume of irradiated tissue, homogeneity of the dose, the size of the daily dose fractions, and the time interval between fractions. ${ }^{2}$

Radiation-induced neurofibroma and de novo development of neurofibroma display the same imaging features, causing a diagnostic dilemma. ${ }^{3}$ Atypical neurofibroma (nuclear hyperchromasia, a moderate number of mitotic figures, and hypercellularity) has been reported and was found to constitute almost all cases. ${ }^{1}$ Our case also demonstrated similar atypical histologic features, showing the presence of poorly 

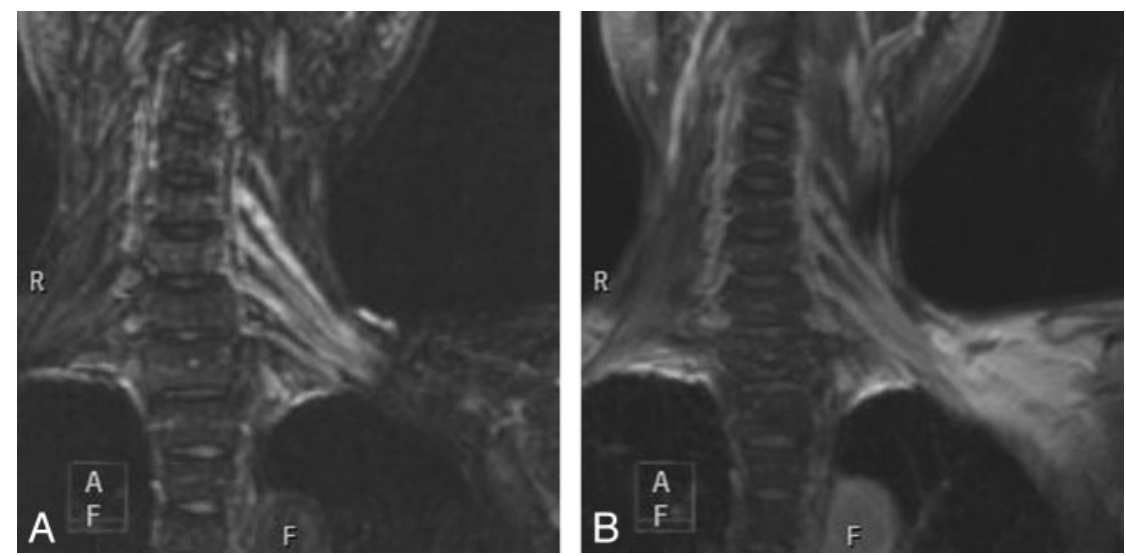

Fig 1. A, Coronal fat-saturated T2-weighted image shows swollen left C4-C8 nerve roots with abnormal hyperintense signals. B, Postcontrast coronal T1-weighted image shows contrast enhancement along the swollen left C4-C8 nerve roots.

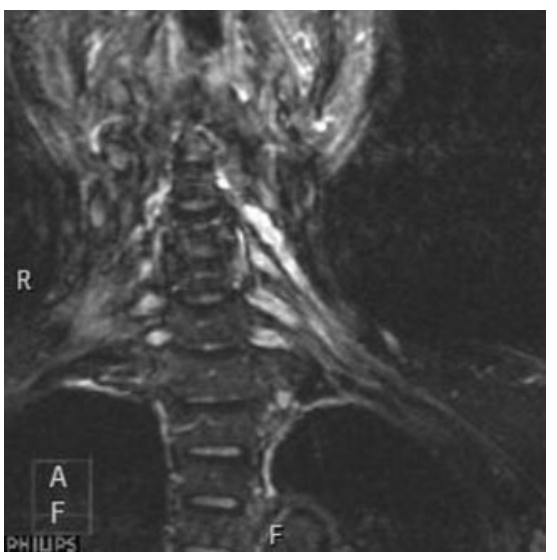

Fig 2. Postcontrast coronal T1-weighted image shows persistent contrast enhancement along the swollen left C4-C8 nerve roots.

demarcated cytoplasm, mild nuclear hyperchromatism, and mitotic activity (Fig 4A). The incidence of such an atypical form progressing into malignancy is not known, due to the rarity of these lesions, but it is expected to be high due to aggressive biologic behavior. This highlights the importance of a thorough histologic examination of any radiation-induced tumor to exclude regions of overt malignant changes. Close and long-term follow-up would also be required be- cause recurrence and malignant transformation are expected to be high. ${ }^{1}$

Our patient had received an "old-fashioned" hypofractionated radiation regime targeting the left neck as adjuvant therapy for left cervical nodal metastasis. Hypofractionated radiation therapy gives larger doses (fractions) of radiation in fewer treatment sessions to avoid intolerable acute toxic effects. The rationale for this approach was the limited machine time in the past as opposed to the "modern" hypofractionated therapy, which has shown promising efficacy, ${ }^{4,5}$ with the ability to conform the radiation beam. Indeed, it has been known for decades from classic radiation therapy that the hypofractionated regime is dangerous. Although the acute toxic effects had been well studied, late toxic effects, in particular the development of new tumor within the irradiated field, remain uncertain due to lack of long-term follow-up for these patients.

Recent literature $^{1}$ review has revealed clinical evidence of radiation in the genesis of peripheral nerve neurofibroma on a dose-dependent basis, typically ranging from 20 to $40 \mathrm{~Gy}$. It has been postulated that 45 Gy of radiation given in a hypofractionated manner is biologically equivalent to $>100 \mathrm{~Gy}$ administered by conventional schedules. ${ }^{4}$ Our patient received a total dose of 39.2 Gy in the left cervical region and developed benign peripheral nerve neurofibromata 11 years later. Although the exact threshold dose is unknown, we are alarmed
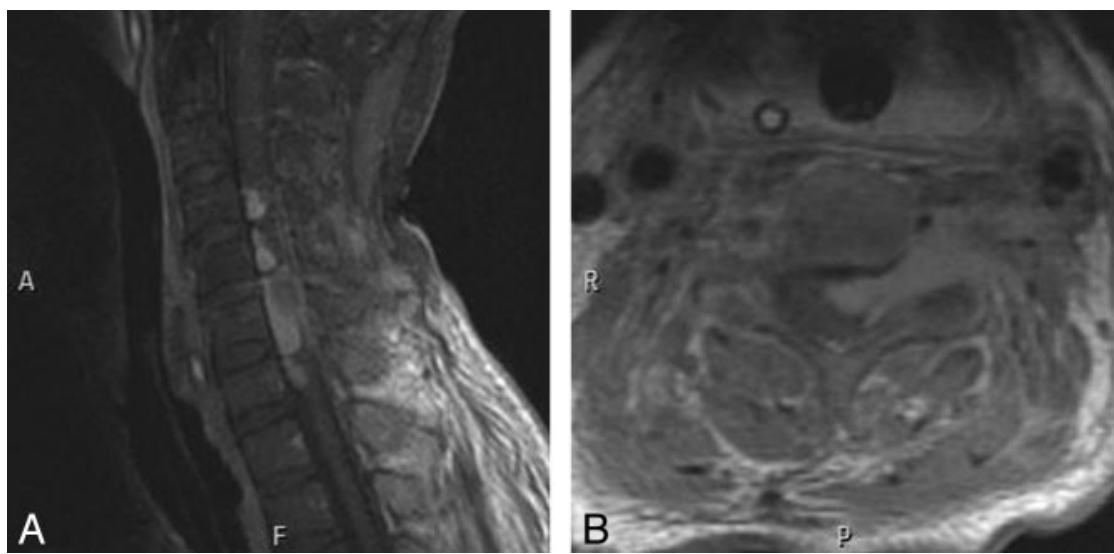

Fig 3. A, Postcontrast sagittal T1-weighted image 16 months after the initial MR imaging study shows a masslike enlargement of the left C4-C8 nerve roots. $B$, Postcontrast axial T1-weighted image shows intraspinal extension with cord compression. 

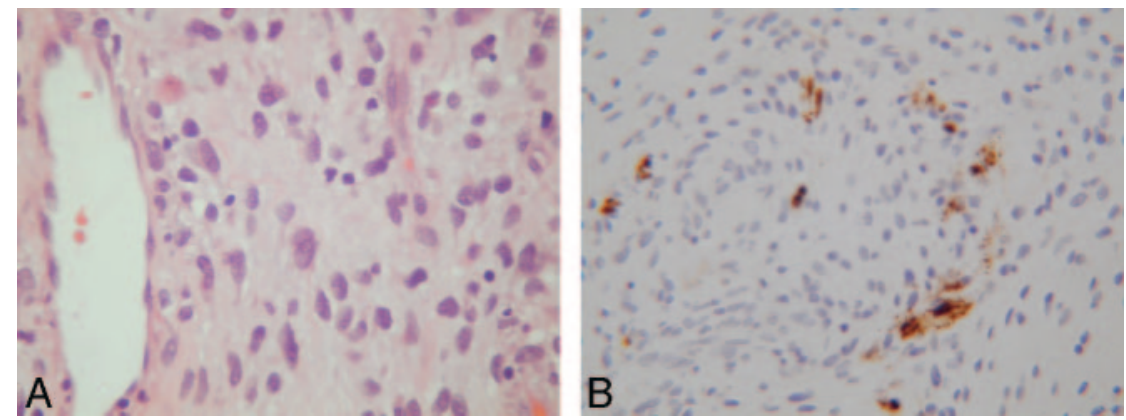

Fig 4. $A$, Photomicrograph shows that the tumor is composed of short spindly tumor cells with poorly demarcated cytoplasm and regular oval nuclei surrounded by myxoid stroma. Some tumor cells show mild nuclear enlargement and hyperchromatism (hematoxylin-eosin, original magnification $\times 200$ ). B, A few of the tumor cells are positive for S100 protein staining (immunohistochemical study).

by present findings that a lower threshold level can induce tumor in any irradiated field due to the increased radiation dose in a hypofractionated regime such as this.

In our patient, serial MR imaging showed persistent swollen nerve roots within the irradiated field with abnormal T2weighted hyperintense signals, mimicking postirradiation neuritis. Not until follow-up scans 16 months later revealed a progressive masslike enlargement of the nerve roots could we derive the correct diagnosis of tumor formation. This enlightened us and stressed the importance of the wide spectrum of late toxic complications of radiation therapy, especially when symptoms persist despite appropriate treatment given, as in our patient, which should alarm enough to search for an alternative diagnosis.

In conclusion, toxicity remains a major concern for patients receiving radiation therapy, especially with the old-fashioned hypofractionated regime, which should not be forgotten, because the life expectancy has increased among these patients. Careful evaluation of late toxicity is essential and should be the next step for future research. With the lack of conclusive data at present, the most difficult challenge is to find the best balance between an optimal tumoricidal dose and an acceptable toxicity rate. Therefore, areas that have been irradiated should be regularly observed both clinically and radiologically, not only for tumor recurrence and other late effects of irradiation but also for the possibility of later development of a new tumor. Moreover, for new tumors, the necessity of systematically searching for previous irradiations in the affected zone should be emphasized. A diligent histologic search for any overt malignant changes is also mandatory.

\section{References}

1. Zadeh G, Buckle C, Shannon P, et al. Radiation-induced peripheral nerve tumors: case series and review of the literature. J Neurooncol 2007;83:205-12

2. Becker M, Schroth G, Zbaren $P$, et al. Long-term changes induced by high-dose irradiation of the head and neck region: imaging findings. Radiographics 1997; 17:5-26

3. Cahan WG, Woodward HQ, Higinbotham NL. Sarcoma arising in irradiated bone. Cancer 1948;1:3-29

4. Nyman J, Johansson K-A, Hulten U. Stereotactic hypofractionated radiotherapy for stage I non-small cell lung cancer: mature results for medically inoperable patients. Lung Cancer 2006;51:97-103. Epub 2005 Oct 4

5. Faria SL, Souhami L, Portelance L, et al. Absence of toxicity with hypofractionated 3-dimensional radiation therapy for inoperable, early stage non-small cell lung cancer. Radiat Oncol 2006;1:42 